George A. Anastassiou (Memphis, TN)

\title{
GENERALIZED $g$-ITERATED FRACTIONAL APPROXIMATIONS BY SUBLINEAR OPERATORS
}

Abstract. We study approximation of functions by sublinear positive operators with applications to several max-product operators under generalized $g$-iterated fractional differentiability. Our work is based on our generalized $g$-iterated fractional results about positive sublinear operators. We produce Jackson type inequalities under iterated initial conditions. Our approach is quantitative by deriving inequalities with right hand sides involving the modulus of continuity of a generalized $g$-iterated fractional derivative of the function being approximated.

1. Background. We study the approximation properties of positive sublinear operators converging to the unit operator related to iterated generalized fractional smoothness of the function being approximated. This is a quantitative study using inequalities.

Definition 1. Set $C_{+}([a, b]):=\left\{f:[a, b] \rightarrow \mathbb{R}_{+}\right.$, continuous functions $\}$. Let $L_{N}: C_{+}([a, b]) \rightarrow C_{+}([a, b])$, for $N \in \mathbb{N}$, be operators such that

$$
L_{N}(\alpha f)=\alpha L_{N}(f), \quad \forall \alpha \geq 0, \forall f \in C_{+}([a, b]) ;
$$

if $f, g \in C_{+}([a, b])$ and $f \leq g$, then

$$
L_{N}(f) \leq L_{N}(g), \quad \forall N \in \mathbb{N} ;
$$

and

$$
L_{N}(f+g) \leq L_{N}(f)+L_{N}(g), \quad \forall f, g \in C_{+}([a, b]) .
$$

We call $\left\{L_{N}\right\}_{N \in \mathbb{N}}$ positive sublinear operators.

2020 Mathematics Subject Classification: 26A33, 41A17, 41A25, 41A36.

Key words and phrases: positive sublinear operators, max-product operators, modulus of continuity, generalized $g$-iterated fractional derivative.

Received 8 November 2019.

Published online 2 November 2020. 
We need a Hölder type inequality:

Theorem 2 (see [2, p. 6]). Let $L: C_{+}([a, b]) \rightarrow C_{+}([a, b])$ be a positive sublinear operator and $f, g \in C_{+}([a, b])$, and let $p, q>1$ with $\frac{1}{p}+\frac{1}{q}=1$. Assume that $L\left((f(\cdot))^{p}\right)\left(s_{*}\right), L\left((g(\cdot))^{q}\right)\left(s_{*}\right)>0$ for some $s_{*} \in[a, b]$. Then

$$
L(f(\cdot) g(\cdot))\left(s_{*}\right) \leq\left(L\left((f(\cdot))^{p}\right)\left(s_{*}\right)\right)^{1 / p}\left(L\left((g(\cdot))^{q}\right)\left(s_{*}\right)\right)^{1 / q} .
$$

Remark 3. By [4, p. 17], if $f, g \in C_{+}([a, b])$, then

$$
\left|L_{N}(f)(x)-L_{N}(g)(x)\right| \leq L_{N}(|f-g|)(x), \quad \forall x \in[a, b] .
$$

Furthermore,

$$
\left|L_{N}(f)(x)-f(x)\right| \leq L_{N}(|f(\cdot)-f(x)|)(x)+|f(x)|\left|L_{N}\left(e_{0}\right)(x)-1\right|
$$

for all $x \in[a, b]$, where $e_{0}(t)=1$.

From now on we assume that $L_{N}(1)=1$. Then

$$
\left|L_{N}(f)(x)-f(x)\right| \leq L_{N}(|f(\cdot)-f(x)|)(x), \quad \forall x \in[a, b] .
$$

In [4, p. 10], the authors introduced the basic max-product Bernstein operators,

$$
B_{N}^{(M)}(f)(x)=\frac{\bigvee_{k=0}^{N} p_{N, k}(x) f(k / N)}{\bigvee_{k=0}^{N} p_{N, k}(x)}, \quad N \in \mathbb{N},
$$

where $\bigvee$ stands for maximum, and $p_{N, k}(x)=\left(\begin{array}{c}N \\ k\end{array}\right) x^{k}(1-x)^{N-k}$ and $f$ : $[0,1] \rightarrow \mathbb{R}_{+}=[0, \infty)$. These are nonlinear and piecewise rational operators, in particular they are positive sublinear operators.

The authors of [4] studied similar such nonlinear operators, including the max-product Favard-Szász-Mirakjan operators and their truncated versions, the max-product Baskakov operators and their truncated versions, and many other similar specific operators. These max-product operators tend to converge faster to the function considered.

We mention from [4, p. 30] that for $f:[0,1] \rightarrow \mathbb{R}_{+}$continuous, we have

$$
\left|B_{N}^{(M)}(f)(x)-f(x)\right| \leq 12 \omega_{1}\left(f, \frac{1}{\sqrt{N+1}}\right) \quad \text { for all } N \in \mathbb{N}, x \in[0,1],
$$

Also from [4, p. 36], for $f:[0,1] \rightarrow \mathbb{R}_{+}$concave we get

$$
\left|B_{N}^{(M)}(f)(x)-f(x)\right| \leq 2 \omega_{1}\left(f, \frac{1}{N}\right) \quad \text { for all } x \in[0,1],
$$

a much faster convergence.

Above, $\omega_{1}$ is the first modulus of continuity. Inequalities (9) and 10 motivate our work. 
Definition 4. For $f \in C([a, b])$, we define

$$
\omega_{1}(f, \delta):=\sup _{\substack{|x-y| \leq \delta \\ x, y \in[a, b]}}|f(x)-f(y)|, \quad \text { where } 0<\delta \leq b-a .
$$

Definition 5 ([1]). Let $\alpha>0$ with $\lceil\alpha\rceil=m$. Let $g \in A C([a, b])$ (absolutely continuous functions) be strictly increasing. Assume $\left(f \circ g^{-1}\right)^{(m)} \circ g$ $\in L_{\infty}([a, b])$. We define the left generalized $g$-fractional derivative of $f$ of order $\alpha$ as follows:

$$
\begin{aligned}
& \left(D_{a+; g}^{\alpha} f\right)(x) \\
& \quad:=\frac{1}{\Gamma(m-\alpha)} \int_{a}^{x}(g(x)-g(t))^{m-\alpha-1} g^{\prime}(t)\left(f \circ g^{-1}\right)^{(m)}(g(t)) d t
\end{aligned}
$$

for all $x \in[a, b]$.

If $\alpha \notin \mathbb{N}$, then by [3, pp. 360-361], we have $D_{a+; g}^{\alpha} f \in C([a, b])$.

We see that

$$
\left(I_{a+; g}^{m-\alpha}\left(\left(f \circ g^{-1}\right)^{(m)} \circ g\right)\right)(x)=\left(D_{a+; g}^{\alpha} f\right)(x), \quad x \geq a .
$$

We set

$$
\begin{aligned}
& D_{a+; g}^{m} f(x):=\left(\left(f \circ g^{-1}\right)^{(m)} \circ g\right)(x), \\
& D_{a+; g}^{0} f(x):=f(x), \quad x \in[a, b] .
\end{aligned}
$$

When $g=\mathrm{id}$, then

$$
D_{a+; g}^{\alpha} f=D_{a+; \mathrm{id}}^{\alpha} f=D_{* a}^{\alpha} f,
$$

the usual left Caputo fractional derivative.

Definition 6 ([1]). Let $g \in A C([a, b])$ be strictly increasing. Assume that $\left(f \circ g^{-1}\right)^{(m)} \circ g \in L_{\infty}([a, b])$, where $N \ni m=\lceil\alpha\rceil, \alpha>0$. We define the right generalized $g$-fractional derivative of $f$ of order $\alpha$ as follows:

$$
\begin{aligned}
& \left(D_{b-; g}^{\alpha} f\right)(x) \\
& \quad:=\frac{(-1)^{m}}{\Gamma(m-\alpha)} \int_{x}^{b}(g(t)-g(x))^{m-\alpha-1} g^{\prime}(t)\left(f \circ g^{-1}\right)^{(m)}(g(t)) d t
\end{aligned}
$$

for all $x \in[a, b]$.

If $\alpha \notin \mathbb{N}$, then by [3, p. 378] we get $\left(D_{b-; g}^{\alpha} f\right) \in C([a, b])$.

We see that

$$
I_{b-; g}^{m-\alpha}\left((-1)^{m}\left(f \circ g^{-1}\right)^{(m)} \circ g\right)(x)=\left(D_{b-; g}^{\alpha} f\right)(x), \quad a \leq x \leq b .
$$

We set

$$
\begin{aligned}
& D_{b-; g}^{m} f(x):=(-1)^{m}\left(\left(f \circ g^{-1}\right)^{(m)} \circ g\right)(x), \\
& D_{b-; g}^{0} f(x):=f(x), \quad x \in[a, b] .
\end{aligned}
$$


If $g=\mathrm{id}$, then

$$
D_{b-; g}^{\alpha} f(x)=D_{b-; i d}^{\alpha} f(x)=D_{b-}^{\alpha} f,
$$

the usual right Caputo fractional derivative.

Denote

$$
D_{b-; g}^{n \alpha}:=D_{b-; g}^{\alpha} D_{b-; g}^{\alpha} \ldots D_{b-; g}^{\alpha} \quad(n \text { times }), n \in \mathbb{N} .
$$

We mention the following $g$-right generalized modified Taylor's formula:

TheOREM 7 ([1]). Suppose that $F_{k}:=D_{b-; g}^{k \alpha} f$ for $k=0,1, \ldots, n+1$ fulfills $F_{k} \circ g^{-1} \in A C([c, d])$, where $c=g(a), d=g(b)$, and $\left(F_{k} \circ g^{-1}\right)^{\prime} \circ g \in$ $L_{\infty}([a, b])$, where $0<\alpha \leq 1$. Then

$$
\begin{aligned}
& f(x)= \sum_{i=0}^{n} \frac{(g(b)-g(x))^{i \alpha}}{\Gamma(i \alpha+1)}\left(D_{b-; g}^{i \alpha} f\right)(b) \\
&+\frac{1}{\Gamma((n+1) \alpha)} \int_{x}^{b}(g(t)-g(x))^{(n+1) \alpha-1} g^{\prime}(t)\left(D_{b-; g}^{(n+1) \alpha} f\right)(t) d t \\
&=\sum_{i=0}^{n} \frac{(g(b)-g(x))^{i \alpha}}{\Gamma(i \alpha+1)}\left(D_{b-; g}^{i \alpha} f\right)(b)+\frac{\left(D_{b-; g}^{(n+1) \alpha} f\right)\left(\psi_{x}\right)}{\Gamma((n+1) \alpha+1)}(g(b)-g(x))^{(n+1) \alpha},
\end{aligned}
$$

where $\psi_{x} \in[x, b]$ for any $x \in[a, b]$.

Denote

$$
D_{a+; g}^{n \alpha}:=D_{a+; g}^{\alpha} D_{a+; g}^{\alpha} \ldots D_{a+; g}^{\alpha} \quad(n \text { times }), n \in \mathbb{N} .
$$

We mention the following $g$-left generalized modified Taylor's formula:

Theorem 8 ([1]). Suppose that $F_{k}^{*}:=D_{a+; g}^{k \alpha} f$ for $k=0,1, \ldots, n+1$ fulfills $F_{k}^{*} \circ g^{-1} \in A C([c, d])$, where $c=g(a), d=g(b)$, and $\left(F_{k}^{*} \circ g^{-1}\right)^{\prime} \circ g \in$ $L_{\infty}([a, b])$, where $0<\alpha \leq 1$. Then

$$
\begin{aligned}
& f(x)= \sum_{i=0}^{n} \frac{(g(x)-g(a))^{i \alpha}}{\Gamma(i \alpha+1)}\left(D_{a+; g}^{i \alpha} f\right)(a) \\
&+\frac{1}{\Gamma((n+1) \alpha)} \int_{a}^{x}(g(x)-g(t))^{(n+1) \alpha-1} g^{\prime}(t)\left(D_{a+; g}^{(n+1) \alpha} f\right)(t) d t \\
&=\sum_{i=0}^{n} \frac{(g(x)-g(a))^{i \alpha}}{\Gamma(i \alpha+1)}\left(D_{a+; g}^{i \alpha} f\right)(a)+\frac{\left(D_{a+; g}^{(n+1) \alpha} f\right)\left(\psi_{x}\right)}{\Gamma((n+1) \alpha+1)}(g(x)-g(a))^{(n+1) \alpha},
\end{aligned}
$$

where $\psi_{x} \in[a, x]$ for any $x \in[a, b]$. 
REMARK 9. Here we restrict ourselves to $0<\alpha<1$. In this case

$$
\begin{aligned}
& \left(D_{a+; g}^{\alpha} f\right)(x)=\frac{1}{\Gamma(1-\alpha)} \int_{a}^{x}(g(x)-g(t))^{-\alpha} g^{\prime}(t)\left(f \circ g^{-1}\right)^{\prime}(g(t)) d t, \\
& \left(D_{b-; g}^{\alpha} f\right)(x)=\frac{-1}{\Gamma(1-\alpha)} \int_{x}^{b}(g(t)-g(x))^{-\alpha} g^{\prime}(t)\left(f \circ g^{-1}\right)^{\prime}(g(t)) d t,
\end{aligned}
$$

for all $x \in[a, b]$.

We observe that

$$
\begin{aligned}
\left|D_{a+; g}^{\alpha} f(x)\right| & \leq \frac{1}{\Gamma(1-\alpha)} \int_{a}^{x}(g(x)-g(t))^{-\alpha} g^{\prime}(t)\left|\left(f \circ g^{-1}\right)^{\prime}(g(t))\right| d t \\
& \leq \frac{\left\|\left(f \circ g^{-1}\right)^{\prime} \circ g\right\|_{\infty,[a, b]}}{\Gamma(1-\alpha)} \int_{a}^{x}(g(x)-g(t))^{-\alpha} g^{\prime}(t) d t \\
& =\frac{\left\|\left(f \circ g^{-1}\right)^{\prime} \circ g\right\|_{\infty,[a, b]}}{\Gamma(1-\alpha)} \frac{(g(x)-g(a))^{1-\alpha}}{1-\alpha} \\
& =\frac{\left\|\left(f \circ g^{-1}\right)^{\prime} \circ g\right\|_{\infty,[a, b]}}{\Gamma(2-\alpha)}(g(x)-g(a))^{1-\alpha} .
\end{aligned}
$$

That is,

$$
\left|\left(D_{a+; g}^{\alpha} f\right)(x)\right| \leq \frac{\left\|\left(f \circ g^{-1}\right)^{\prime} \circ g\right\|_{\infty,[a, b]}}{\Gamma(2-\alpha)}(g(x)-g(a))^{1-\alpha}<+\infty
$$

for all $x \in[a, b]$.

Clearly $\left(D_{a+; g}^{\alpha} f\right)(a)=0$. That is,

$$
\left(D_{x_{0}+; g}^{\alpha} f\right)\left(x_{0}\right)=0, \quad \forall x_{0} \in[a, b] .
$$

We define

$$
\left(D_{x_{0}+; g}^{\alpha} f\right)(x)=0 \quad \text { for } a \leq x<x_{0} .
$$

Similarly we get (by [5, p. 107])

$$
\begin{aligned}
\left|D_{b-; g}^{\alpha} f(x)\right| & \leq \frac{1}{\Gamma(1-\alpha)} \int_{x}^{b}(g(t)-g(x))^{-\alpha} g^{\prime}(t)\left|\left(f \circ g^{-1}\right)^{\prime}(g(t))\right| d t \\
& \leq \frac{\left\|\left(f \circ g^{-1}\right)^{\prime} \circ g\right\|_{\infty,[a, b]}}{\Gamma(1-\alpha)} \int_{x}^{b}(g(t)-g(x))^{-\alpha} g^{\prime}(t) d t \\
& =\frac{\left\|\left(f \circ g^{-1}\right)^{\prime} \circ g\right\|_{\infty,[a, b]}}{\Gamma(2-\alpha)}(g(b)-g(x))^{1-\alpha} .
\end{aligned}
$$


That is,

$$
\left|D_{b-; g}^{\alpha} f(x)\right| \leq \frac{\left\|\left(f \circ g^{-1}\right)^{\prime} \circ g\right\|_{\infty,[a, b]}}{\Gamma(2-\alpha)}(g(b)-g(x))^{1-\alpha}<+\infty
$$

for all $x \in[a, b]$.

Clearly $\left(D_{b-; g}^{\alpha} f\right)(b)=0$. In particular,

$$
\left(D_{x_{0}-; g}^{\alpha} f\right)\left(x_{0}\right)=0, \quad \forall x_{0} \in[a, b] .
$$

We define

$$
\left(D_{x_{0}-; g}^{\alpha} f\right)(x)=0 \quad \text { for } x_{0}<x \leq b .
$$

From Theorem 7 we have

THEOREM 10. Let $0<\alpha<1$, and let $g \in C^{1}([a, b])$ be strictly increasing, so that $g^{-1} \in C^{1}([g(a), g(b)])$. Assume that $F_{k}:=D_{x_{0}-; g}^{k \alpha} f$ for $k=0,1, \ldots, n+1$ fulfills $F_{k} \in C^{1}\left(\left[a, x_{0}\right]\right)$, where $x_{0} \in[a, b]$ is fixed. Then

$$
\begin{aligned}
f(x)-f\left(x_{0}\right) & =\sum_{i=2}^{n} \frac{\left(g\left(x_{0}\right)-g(x)\right)^{i \alpha}}{\Gamma(i \alpha+1)}\left(D_{x_{0}-; g}^{i \alpha} f\right)\left(x_{0}\right) \\
& +\frac{1}{\Gamma((n+1) \alpha)} \int_{x}^{x_{0}}(g(t)-g(x))^{(n+1) \alpha-1} g^{\prime}(t)\left(D_{x_{0}-; g}^{(n+1) \alpha} f\right)(t) d t
\end{aligned}
$$

for all $x \in\left[a, x_{0}\right]$.

From Theorem 8 we have

TheOREM 11. Let $0<\alpha<1, g \in C^{1}([a, b])$ and strictly increasing, so that $g^{-1} \in C^{1}([g(a), g(b)])$. Assume that $F_{k}^{*}:=D_{x_{0}+; g}^{k \alpha}$ f for $k=0,1, \ldots, n+1$ fulfills $F_{k}^{*} \in C^{1}\left(\left[x_{0}, b\right]\right)$, where $x_{0} \in[a, b]$ is fixed. Then

$$
\begin{aligned}
f(x)-f\left(x_{0}\right) & =\sum_{i=2}^{n} \frac{\left(g(x)-g\left(x_{0}\right)\right)^{i \alpha}}{\Gamma(i \alpha+1)}\left(D_{x_{0}+; g}^{i \alpha} f\right)\left(x_{0}\right) \\
& +\frac{1}{\Gamma((n+1) \alpha)} \int_{x_{0}}^{x}(g(x)-g(t))^{(n+1) \alpha-1} g^{\prime}(t)\left(D_{x_{0}+; g}^{(n+1) \alpha} f\right)(t) d t
\end{aligned}
$$

for all $x \in\left[x_{0}, b\right]$.

REMARK 12. If in Theorem 10, $\left(D_{x_{0}-; g}^{i \alpha} f\right)\left(x_{0}\right)=0$ for $i=2, \ldots, n$, then

$$
\begin{aligned}
f(x) & -f\left(x_{0}\right) \\
& =\frac{1}{\Gamma((n+1) \alpha)} \int_{x}^{x_{0}}(g(t)-g(x))^{(n+1) \alpha-1} g^{\prime}(t)\left(D_{x_{0}-; g}^{(n+1) \alpha} f\right)(t) d t
\end{aligned}
$$

for all $x \in\left[a, x_{0}\right]$. 
Next, if in Theorem 11. $\left(D_{x_{0}+; g}^{i \alpha} f\right)\left(x_{0}\right)=0$ for $i=2, \ldots, n$, then

$$
\begin{aligned}
f(x) & -f\left(x_{0}\right) \\
& =\frac{1}{\Gamma((n+1) \alpha)} \int_{x_{0}}^{x}(g(x)-g(t))^{(n+1) \alpha-1} g^{\prime}(t)\left(D_{x_{0}+; g}^{(n+1) \alpha} f\right)(t) d t
\end{aligned}
$$

for all $x \in\left[x_{0}, b\right]$.

Definition 13. Let $D_{x_{0} ; g}^{(n+1) \alpha} f$ denote any of $D_{x_{0}-; g}^{(n+1) \alpha} f, D_{x_{0}+; g}^{(n+1) \alpha} f, n \in \mathbb{N}$, and let $\delta>0$. We set

$$
\omega_{1}\left(\left(D_{x_{0} ; g}^{(n+1) \alpha} f\right) \circ g^{-1}, \delta\right)
$$

$:=\max \left\{\omega_{1}\left(\left(D_{x_{0} ; g}^{(n+1) \alpha} f\right) \circ g^{-1}, \delta\right)_{\left[g(a), g\left(x_{0}\right)\right]}, \omega_{1}\left(\left(D_{x_{0}+; g}^{(n+1) \alpha} f\right) \circ g^{-1}, \delta\right)_{\left[g\left(x_{0}\right), g(b)\right]}\right\}$, where $x_{0} \in[a, b]$. Here the moduli of continuity are considered over $\left[g(a), g\left(x_{0}\right)\right]$ and $\left[g\left(x_{0}\right), g(b)\right]$, respectively.

2. Main results. We will use the following important result.

Theorem 14. Let $0<\alpha<1, f, g \in C^{1}([a, b])$, where $g$ is strictly increasing and $g^{-1} \in C^{1}([g(a), g(b)])$. Assume that $D_{x_{0}-; g}^{k \alpha} f \in C^{1}\left(\left[a, x_{0}\right]\right)$ and $D_{x_{0}+; g}^{k \alpha} f \in C^{1}\left(\left[x_{0}, b\right]\right)$ for $k=1, \ldots, n+1$, where $x_{0} \in[a, b]$ is fixed. Further assume that $\left(D_{x_{0} \pm ; g}^{i \alpha} f\right)\left(x_{0}\right)=0, i=2, \ldots, n+1$. Then

$$
\begin{aligned}
\left|f(x)-f\left(x_{0}\right)\right| \leq & \frac{\omega_{1}\left(\left(D_{x_{0} ; g}^{(n+1) \alpha} f\right) \circ g^{-1}, \delta\right)}{\Gamma((n+1) \alpha+1)} \\
& \times\left[\left|g(x)-g\left(x_{0}\right)\right|^{(n+1) \alpha}+\frac{\left|g(x)-g\left(x_{0}\right)\right|^{(n+1) \alpha+1}}{\delta((n+1) \alpha+1)}\right]
\end{aligned}
$$

for all $x \in[a, b], \delta>0$.

Proof. By $\left(D_{x_{0}+; g}^{i \alpha} f\right)\left(x_{0}\right)=0$ for $i=2, \ldots, n+1$, we have

$$
\begin{aligned}
f(x)-f\left(x_{0}\right)= & \frac{1}{\Gamma((n+1) \alpha)} \int_{x_{0}}^{x}(g(x)-g(t))^{(n+1) \alpha-1} g^{\prime}(t) \\
& \times\left[\left(D_{x_{0}+; g}^{(n+1) \alpha} f\right)(t)-\left(D_{x_{0}+; g}^{(n+1) \alpha} f\right)\left(x_{0}\right)\right] d t
\end{aligned}
$$

for all $x \in\left[x_{0}, b\right]$. Hence $(z:=g(t))$

$$
\begin{aligned}
f(x)-f\left(x_{0}\right) & =\frac{1}{\Gamma((n+1) \alpha)} \int_{g\left(x_{0}\right)}^{g(x)}(g(x)-z)^{(n+1) \alpha-1} \\
\times & {\left[\left(\left(D_{x_{0}+; g}^{(n+1) \alpha} f\right) \circ g^{-1}\right)(z)-\left(\left(D_{x_{0}+; g}^{(n+1) \alpha} f\right) \circ g^{-1}\right)\left(g\left(x_{0}\right)\right)\right] d z }
\end{aligned}
$$

for all $x \in\left[x_{0}, b\right]$. 
By $\left(D_{x_{0}-; g}^{i \alpha} f\right)\left(x_{0}\right)=0$ for $i=2, \ldots, n+1$, we have

$$
\begin{aligned}
f(x)-f\left(x_{0}\right)= & \frac{1}{\Gamma((n+1) \alpha)} \int_{x}^{x_{0}}(g(t)-g(x))^{(n+1) \alpha-1} g^{\prime}(t) \\
& \times\left[\left(D_{x_{0}-; g}^{(n+1) \alpha} f\right)(t)-\left(D_{x_{0}-; g}^{(n+1) \alpha} f\right)\left(x_{0}\right)\right] d t
\end{aligned}
$$

for all $x \in\left[a, x_{0}\right]$. Hence $(z:=g(t))$

$$
\begin{aligned}
& f(x)-f\left(x_{0}\right)=\frac{1}{\Gamma((n+1) \alpha)} \int_{g(x)}^{g\left(x_{0}\right)}(z-g(x))^{(n+1) \alpha-1} \\
& \times\left[\left(\left(D_{x_{0}-; g}^{(n+1) \alpha} f\right) \circ g^{-1}\right)(z)-\left(\left(D_{x_{0}-; g}^{(n+1) \alpha} f\right) \circ g^{-1}\right)\left(g\left(x_{0}\right)\right)\right] d z
\end{aligned}
$$

for all $x \in\left[a, x_{0}\right]$. We find that $\left(x_{0} \leq x \leq b\right)$

$$
\left|f(x)-f\left(x_{0}\right)\right| \leq \frac{1}{\Gamma((n+1) \alpha)} \int_{g\left(x_{0}\right)}^{g(x)}(g(x)-z)^{(n+1) \alpha-1}
$$$$
\times\left|\left(\left(D_{x_{0}+; g}^{(n+1) \alpha} f\right) \circ g^{-1}\right)(z)-\left(\left(D_{x_{0}+; g}^{(n+1) \alpha} f\right) \circ g^{-1}\right)\left(g\left(x_{0}\right)\right)\right| d z
$$

$\underset{\left(\delta_{1}>0\right)}{\leq} \frac{1}{\Gamma((n+1) \alpha)} \int_{g\left(x_{0}\right)}^{g(x)}(g(x)-z)^{(n+1) \alpha-1}$

$$
\begin{aligned}
& \times \omega_{1}\left(\left(D_{x_{0}+; g}^{(n+1) \alpha} f\right) \circ g^{-1}, \frac{\delta_{1}\left|z-g\left(x_{0}\right)\right|}{\delta_{1}}\right)_{\left[g\left(x_{0}\right), g(b)\right]} d z \\
\leq & \frac{\omega_{1}\left(\left(D_{x_{0}+; g}^{(n+1) \alpha} f\right) \circ g^{-1}, \delta_{1}\right)_{\left[g\left(x_{0}\right), g(b)\right]}}{\Gamma((n+1) \alpha)} \\
& \times \int_{g\left(x_{0}\right)}^{g(x)}(g(x)-z)^{(n+1) \alpha-1}\left(1+\frac{z-g\left(x_{0}\right)}{\delta_{1}}\right) d z \\
= & \frac{\omega_{1}\left(\left(D_{x_{0}+; g}^{(n+1) \alpha} f\right) \circ g^{-1}, \delta_{1}\right)_{\left[g\left(x_{0}\right), g(b)\right]}}{\Gamma((n+1) \alpha)} \\
& \times\left[\frac{\left(g(x)-g\left(x_{0}\right)\right)^{(n+1) \alpha}}{(n+1) \alpha}+\frac{1}{\delta_{1}} \int_{g\left(x_{0}\right)}^{g(x)}(g(x)-z)^{(n+1) \alpha-1}\left(z-g\left(x_{0}\right)\right)^{2-1} d z\right] \\
= & \frac{\omega_{1}\left(\left(D_{x_{0}+; g}^{(n+1) \alpha} f\right) \circ g^{-1}, \delta_{1}\right)_{\left[g\left(x_{0}\right), g(b)\right]}}{\Gamma((n+1) \alpha)} \\
& \times\left[\frac{\left(g(x)-g\left(x_{0}\right)\right)^{(n+1) \alpha}}{(n+1) \alpha}+\frac{1}{\delta_{1}} \frac{\Gamma((n+1) \alpha) \Gamma(2)}{\Gamma((n+1) \alpha+2)}\left(g(x)-g\left(x_{0}\right)\right)^{(n+1) \alpha+1}\right]
\end{aligned}
$$




$$
\begin{aligned}
= & \frac{\omega_{1}\left(\left(D_{x_{0}+; g}^{(n+1) \alpha} f\right) \circ g^{-1}, \delta_{1}\right)\left[g\left(x_{0}\right), g(b)\right]}{\Gamma((n+1) \alpha)} \\
& \times\left[\frac{\left(g(x)-g\left(x_{0}\right)\right)^{(n+1) \alpha}}{(n+1) \alpha}+\frac{1}{\delta_{1}} \frac{\left(g(x)-g\left(x_{0}\right)\right)^{(n+1) \alpha+1}}{(n+1) \alpha((n+1) \alpha+1)}\right] .
\end{aligned}
$$

We have proved that

$$
\begin{aligned}
\left|f(x)-f\left(x_{0}\right)\right| \leq & \frac{\omega_{1}\left(\left(D_{x_{0}+; g}^{(n+1) \alpha} f\right) \circ g^{-1}, \delta_{1}\right)_{\left[g\left(x_{0}\right), g(b)\right]}}{\Gamma((n+1) \alpha+1)} \\
& \times\left[\left(g(x)-g\left(x_{0}\right)\right)^{(n+1) \alpha}+\frac{\left(g(x)-g\left(x_{0}\right)\right)^{(n+1) \alpha+1}}{\delta_{1}((n+1) \alpha+1)}\right]
\end{aligned}
$$

for all $x \in\left[x_{0}, b\right], \delta_{1}>0$.

We deduce that $\left(a \leq x \leq x_{0}\right)$

$$
\begin{aligned}
& \left|f(x)-f\left(x_{0}\right)\right| \stackrel{42}{\leq} \frac{1}{\Gamma((n+1) \alpha)} \int_{g(x)}^{g\left(x_{0}\right)}(z-g(x))^{(n+1) \alpha-1} \\
& \times\left|\left(\left(D_{x_{0}-; g}^{(n+1) \alpha} f\right) \circ g^{-1}\right)(z)-\left(\left(D_{x_{0}-; g}^{(n+1) \alpha} f\right) \circ g^{-1}\right)\left(g\left(x_{0}\right)\right)\right| d z \\
& \underset{\left(\delta_{2}>0\right)}{\leq} \frac{1}{\Gamma((n+1) \alpha)} \int_{g(x)}^{g\left(x_{0}\right)}(z-g(x))^{(n+1) \alpha-1}
\end{aligned}
$$$$
\times \omega_{1}\left(\left(D_{x_{0}-; g}^{(n+1) \alpha} f\right) \circ g^{-1}, \frac{\delta_{2}\left|z-g\left(x_{0}\right)\right|}{\delta_{2}}\right)_{\left[g(a), g\left(x_{0}\right)\right]} d z
$$$$
\leq \frac{\omega_{1}\left(\left(D_{x_{0}-; g}^{(n+1) \alpha} f\right) \circ g^{-1}, \delta_{2}\right)_{\left[g(a), g\left(x_{0}\right)\right]}}{\Gamma((n+1) \alpha)}
$$$$
\times \int_{g(x)}^{g\left(x_{0}\right)}(z-g(x))^{(n+1) \alpha-1}\left(1+\frac{g\left(x_{0}\right)-z}{\delta_{2}}\right) d z
$$$$
=\frac{\omega_{1}\left(\left(D_{x_{0}-; g}^{(n+1) \alpha} f\right) \circ g^{-1}, \delta_{2}\right)_{\left[g(a), g\left(x_{0}\right)\right]}}{\Gamma((n+1) \alpha)}
$$$$
\times\left[\frac{\left(g\left(x_{0}\right)-g(x)\right)^{(n+1) \alpha}}{(n+1) \alpha}+\frac{1}{\delta_{2}} \int_{g(x)}^{g\left(x_{0}\right)}\left(g\left(x_{0}\right)-z\right)^{2-1}(z-g(x))^{(n+1) \alpha-1} d z\right]
$$

$$
\begin{aligned}
= & \frac{\omega_{1}\left(\left(D_{x_{0}-; g}^{(n+1) \alpha} f\right) \circ g^{-1}, \delta_{2}\right)_{\left[g(a), g\left(x_{0}\right)\right]}}{\Gamma((n+1) \alpha)} \\
& \times\left[\frac{\left(g\left(x_{0}\right)-g(x)\right)^{(n+1) \alpha}}{(n+1) \alpha}+\frac{1}{\delta_{2}} \frac{\Gamma(2) \Gamma((n+1) \alpha)}{\Gamma((n+1) \alpha+2)}\left(g\left(x_{0}\right)-g(x)\right)^{(n+1) \alpha+1}\right]
\end{aligned}
$$




$$
\begin{aligned}
= & \frac{\omega_{1}\left(\left(D_{x_{0}-; g}^{(n+1) \alpha} f\right) \circ g^{-1}, \delta_{2}\right)\left[g(a), g\left(x_{0}\right)\right]}{\Gamma((n+1) \alpha)} \\
& \times\left[\frac{\left(g\left(x_{0}\right)-g(x)\right)^{(n+1) \alpha}}{(n+1) \alpha}+\frac{1}{\delta_{2}} \frac{\left(g\left(x_{0}\right)-g(x)\right)^{(n+1) \alpha+1}}{(n+1) \alpha((n+1) \alpha+1)}\right] \\
= & \frac{\omega_{1}\left(\left(D_{x_{0}-; g}^{(n+1) \alpha} f\right) \circ g^{-1}, \delta_{2}\right)\left[g(a), g\left(x_{0}\right)\right]}{\Gamma((n+1) \alpha+1)} \\
& \times\left[\left(g\left(x_{0}\right)-g(x)\right)^{(n+1) \alpha}+\frac{\left(g\left(x_{0}\right)-g(x)\right)^{(n+1) \alpha+1}}{\delta_{2}((n+1) \alpha+1)}\right] .
\end{aligned}
$$

We have proved that

$$
\begin{aligned}
\left|f(x)-f\left(x_{0}\right)\right| \leq & \frac{\omega_{1}\left(\left(D_{x_{0}-; g}^{(n+1) \alpha} f\right) \circ g^{-1}, \delta_{2}\right)\left[g(a), g\left(x_{0}\right)\right]}{\Gamma((n+1) \alpha+1)} \\
& \times\left[\left(g\left(x_{0}\right)-g(x)\right)^{(n+1) \alpha}+\frac{\left(g\left(x_{0}\right)-g(x)\right)^{(n+1) \alpha+1}}{\delta_{2}((n+1) \alpha+1)}\right]
\end{aligned}
$$

for all $x \in\left[a, x_{0}\right], \delta_{2}>0$.

By (45) and (47), setting $\delta=\delta_{1}=\delta_{2}>0$, we derive (39).

Corollary 15 (to Theorem 14). Under the assumptions of Theorem 14 .

$$
\begin{aligned}
\left|f(\cdot)-f\left(x_{0}\right)\right| \leq & \frac{\omega_{1}\left(\left(D_{x_{0} ; g}^{(n+1) \alpha} f\right) \circ g^{-1}, \delta\right)}{\Gamma((n+1) \alpha+1)} \\
& \times\left[\left|g(\cdot)-g\left(x_{0}\right)\right|^{(n+1) \alpha}+\frac{\left|g(\cdot)-g\left(x_{0}\right)\right|^{(n+1) \alpha+1}}{\delta((n+1) \alpha+1)}\right]
\end{aligned}
$$

for $\delta>0$, valid on the interval $[a, b]$.

Our first result on approximation by positive sublinear operators follows:

Theorem 16. Suppose $0<\alpha<1, f \in C_{+}([a, b]), f, g \in C^{1}([a, b])$, $g$ is strictly increasing and $g^{-1} \in C^{1}([g(a), g(b)])$. Assume that $D_{x_{0}-; g}^{k \alpha} f \in$ $C^{1}\left(\left[a, x_{0}\right]\right)$ and $D_{x_{0}+; g}^{k \alpha} f \in C^{1}\left(\left[x_{0}, b\right]\right)$, for $k=1, \ldots, n+1$, where $x_{0} \in[a, b]$ is fixed. Further assume that $\left(D_{x_{0} \pm ;}^{i \alpha} f\right)\left(x_{0}\right)=0, i=2, \ldots, n+1$. Let $L_{N}$ : $C_{+}([a, b]) \rightarrow C_{+}([a, b])$, for all $N \in \mathbb{N}$, be positive sublinear operators such that $L_{N}(1)=1$ for all $N \in \mathbb{N}$. Then

$$
\begin{aligned}
& \left|L_{N}(f)\left(x_{0}\right)-f\left(x_{0}\right)\right| \leq \frac{\omega_{1}\left(\left(D_{x_{0} ; g}^{(n+1) \alpha} f\right) \circ g^{-1}, \delta\right)}{\Gamma((n+1) \alpha+1)} \\
& \quad \times\left[L_{N}\left(\left|g(\cdot)-g\left(x_{0}\right)\right|^{(n+1) \alpha}\right)\left(x_{0}\right)+\frac{L_{N}\left(\left|g(\cdot)-g\left(x_{0}\right)\right|^{(n+1) \alpha+1}\right)\left(x_{0}\right)}{\delta((n+1) \alpha+1)}\right]
\end{aligned}
$$

for all $N \in \mathbb{N}$ and $\delta>0$. 
Proof. By (7) and 48 we have

$$
\begin{aligned}
& \leq \frac{\omega_{1}\left(\left(D_{x_{0} ; g}^{(n+1) \alpha} f\right) \circ g^{-1}, \delta\right)}{\Gamma((n+1) \alpha+1)} \\
& \times\left[L_{N}\left(\left|g(\cdot)-g\left(x_{0}\right)\right|^{(n+1) \alpha}\right)\left(x_{0}\right)+\frac{L_{N}\left(\left|g(\cdot)-g\left(x_{0}\right)\right|^{(n+1) \alpha+1}\right)\left(x_{0}\right)}{\delta((n+1) \alpha+1)}\right], \quad \delta>0,
\end{aligned}
$$

proving the claim.

THEOREM 17. Under the assumptions of Theorem 16, assume moreover that $L_{N}\left(\left|g(\cdot)-g\left(x_{0}\right)\right|^{(n+1) \alpha+1}\right)\left(x_{0}\right)>0$ for all $N \in \mathbb{N}$. Then

$$
\begin{aligned}
& \left|L_{N}(f)\left(x_{0}\right)-f(x)\right| \leq \frac{(n+1) \alpha+2}{\Gamma((n+1) \alpha+2)} \\
& \quad \times \omega_{1}\left(\left(D_{x_{0} ; g}^{(n+1) \alpha} f\right) \circ g^{-1},\left(L_{N}\left(\left|g(\cdot)-g\left(x_{0}\right)\right|^{(n+1) \alpha+1}\right)\left(x_{0}\right)\right)^{\frac{1}{(n+1) \alpha+1}}\right) \\
& \quad \times\left(L_{N}\left(\left|g(\cdot)-g\left(x_{0}\right)\right|^{(n+1) \alpha+1}\right)\left(x_{0}\right)\right)^{\frac{(n+1) \alpha}{(n+1) \alpha+1}}
\end{aligned}
$$

for all $N \in \mathbb{N}$.

Proof. By Theorem 2 (see (4)), we get

$$
\begin{aligned}
L_{N}\left(\left|g(\cdot)-g\left(x_{0}\right)\right|^{(n+1) \alpha}\right. & \left(x_{0}\right) \\
& \leq\left(L_{N}\left(\left|g(\cdot)-g\left(x_{0}\right)\right|^{(n+1) \alpha+1}\right)\left(x_{0}\right)\right)^{\frac{(n+1) \alpha}{(n+1) \alpha+1}} .
\end{aligned}
$$

Choose

$$
\delta:=\left(L_{N}\left(\left|g(\cdot)-g\left(x_{0}\right)\right|^{(n+1) \alpha+1}\right)\left(x_{0}\right)\right)^{\frac{1}{(n+1) \alpha+1}}>0,
$$

i.e.

$$
\delta^{(n+1) \alpha+1}=L_{N}\left(\left|g(\cdot)-g\left(x_{0}\right)\right|^{(n+1) \alpha+1}\right)\left(x_{0}\right) .
$$

By 49) we obtain

$$
\begin{aligned}
& \left|L_{N}(f)\left(x_{0}\right)-f\left(x_{0}\right)\right| \\
& \leq \frac{\omega_{1}\left(\left(D_{x_{0} ; g}^{(n+1) \alpha} f\right) \circ g^{-1},\left(L_{N}\left(\left|g(\cdot)-g\left(x_{0}\right)\right|^{(n+1) \alpha+1}\right)\left(x_{0}\right)\right)^{\frac{1}{(n+1) \alpha+1}}\right)}{\Gamma((n+1) \alpha+1)} \\
& \quad \times\left[\left(L_{N}\left(\left|g(\cdot)-g\left(x_{0}\right)\right|^{(n+1) \alpha+1}\right)\left(x_{0}\right)\right)^{\frac{(n+1) \alpha}{(n+1) \alpha+1}}+\frac{\delta^{(n+1) \alpha+1}}{\delta((n+1) \alpha+1)}\right]
\end{aligned}
$$




$$
\begin{aligned}
= & \frac{\omega_{1}\left(\left(D_{x_{0} ; g}^{(n+1) \alpha} f\right) \circ g^{-1},\left(L_{N}\left(\left|g(\cdot)-g\left(x_{0}\right)\right|^{(n+1) \alpha+1}\right)\left(x_{0}\right)\right)^{\frac{1}{(n+1) \alpha+1}}\right)}{\Gamma((n+1) \alpha+1)} \\
& \times\left[\left(L_{N}\left(\left|g(\cdot)-g\left(x_{0}\right)\right|^{(n+1) \alpha+1}\right)\left(x_{0}\right)\right)^{\frac{(n+1) \alpha}{(n+1) \alpha+1}}\right. \\
& \left.+\frac{\left(L_{N}\left(\left|g(\cdot)-g\left(x_{0}\right)\right|^{(n+1) \alpha+1}\right)\left(x_{0}\right)\right)^{\frac{(n+1) \alpha}{(n+1) \alpha+1}}}{((n+1) \alpha+1)}\right] \\
= & \frac{\omega_{1}\left(\left(D_{x_{0} ; g}^{(n+1) \alpha} f\right) \circ g^{-1},\left(L_{N}\left(\left|g(\cdot)-g\left(x_{0}\right)\right|^{(n+1) \alpha+1}\right)\left(x_{0}\right)\right)^{\frac{1}{(n+1) \alpha+1}}\right)}{\Gamma((n+1) \alpha+1)} \\
& \times\left(L_{N}\left(\left|g(\cdot)-g\left(x_{0}\right)\right|^{(n+1) \alpha+1}\right)\left(x_{0}\right)\right)^{\frac{(n+1) \alpha}{(n+1) \alpha+1}}\left[1+\frac{1}{(n+1) \alpha+1}\right] \\
= & \frac{((n+1) \alpha+2)}{\Gamma((n+1) \alpha+2)} \\
& \times \omega_{1}\left(\left(D_{x_{0} ; g}^{(n+1) \alpha} f\right) \circ g^{-1},\left(L_{N}\left(\left|g(\cdot)-g\left(x_{0}\right)\right|^{(n+1) \alpha+1}\right)\left(x_{0}\right)\right)^{\frac{(n+1) \alpha+1}{(n+1) \alpha}}\right.
\end{aligned}
$$

proving (51).

REMARK 18. In the setting of Theorem 17, suppose moreover that $\left(L_{N}\left(\left|g(\cdot)-g\left(x_{0}\right)\right|^{(n+1) \alpha+1}\right)\left(x_{0}\right)\right) \rightarrow 0$. Then $L_{N}(f)\left(x_{0}\right) \rightarrow f\left(x_{0}\right)$ as $N \rightarrow+\infty$.

Corollary 19. In the setting of Theorem 16, assume moreover that $L_{N}\left(\left|\cdot-x_{0}\right|^{(n+1) \alpha+1}\right)\left(x_{0}\right)>0$ for all $N \in \mathbb{N}$. Then

$$
\begin{aligned}
& \left|L_{N}(f)\left(x_{0}\right)-f\left(x_{0}\right)\right| \leq \frac{(n+1) \alpha+2}{\Gamma((n+1) \alpha+2)} \\
& \times \omega_{1}\left(\left(D_{x_{0} ; g}^{(n+1) \alpha} f\right) \circ g^{-1},\left\|g^{\prime}\right\|_{\infty,[a, b]}\left(L_{N}\left(\left|\cdot-x_{0}\right|^{(n+1) \alpha+1}\right)\left(x_{0}\right)\right)^{\frac{1}{(n+1) \alpha+1}}\right) \\
& \times\left\|g^{\prime}\right\|_{\infty,[a, b]}^{(n+1) \alpha}\left(L_{N}\left(\left|\cdot-x_{0}\right|^{(n+1) \alpha+1}\right)\left(x_{0}\right)\right)^{\frac{(n+1) \alpha}{(n+1) \alpha+1}}
\end{aligned}
$$

for all $N \in \mathbb{N}$.

Proof. Use Theorem 17 (see (51)), the properties of $\omega_{1}$ and $L_{N}$, and the fact that $\left|g(x)-g\left(x_{0}\right)\right| \leq\left\|g^{\prime}\right\|_{\infty,[a, b]}\left|x-x_{0}\right|$, along with $\left|g(x)-g\left(x_{0}\right)\right| \geq$ (inf $\left._{x \in[a, b \mid}\left|g^{\prime}(x)\right|\right)\left|x-x_{0}\right|$ for all $x, x_{0} \in[a, b]$, since $g^{\prime}(x)>0$ for all $x \in[a, b]$. Clearly 55$)$ is then valid, and $L_{N}\left(\left|g(\cdot)-g\left(x_{0}\right)\right|^{(n+1) \alpha+1}\right)\left(x_{0}\right)>0$ for all $N \in \mathbb{N}$.

\section{Applications}

3.1. Case $0<(n+1) \alpha<1$. Here we apply Corollary 19 to well known max-product operators. 
REMARK 20. The max-product Bernstein operators $B_{N}^{(M)}(f)(x)$ are defined by (8) (see also [4, p. 10]); here $f:[0,1] \rightarrow \mathbb{R}_{+}$is a continuous function.

We have $B_{N}^{(M)}(1)=1$, and

$$
B_{N}^{(M)}(|\cdot-x|)(x) \leq \frac{6}{\sqrt{N+1}}, \quad \forall x \in[0,1], \forall N \in \mathbb{N}
$$

(see [4, p. 31]).

$B_{N}^{(M)}$ are positive sublinear operators and thus they possess the monotonicity property; also since $|\cdot-x| \leq 1$, we have $|\cdot-x|^{\beta} \leq 1$ for all $x \in[0,1]$ and all $\beta>0$.

Therefore

$$
B_{N}^{(M)}\left(|\cdot-x|^{1+\beta}\right)(x) \leq \frac{6}{\sqrt{N+1}}, \quad \forall x \in[0,1], \forall N \in \mathbb{N}, \forall \beta>0 .
$$

Furthermore, clearly

$$
B_{N}^{(M)}\left(|\cdot-x|^{1+\beta}\right)(x)>0, \quad \forall N \in \mathbb{N}, \forall \beta \geq 0, \forall x \in(0,1) .
$$

The operator $B_{N}^{(M)}$ maps $C_{+}([0,1])$ into itself.

Theorem 21. Let $0<\alpha<1, f \in C_{+}([0,1]), f, g \in C^{1}([0,1])$, where $g$ is strictly increasing and $g^{-1} \in C^{1}([g(0), g(1)])$. Assume that $D_{x-; g}^{k \alpha} f \in$ $C^{1}([0, x])$ and $D_{x+; g}^{k \alpha} f \in C^{1}([x, 1])$ for $k=1, \ldots, n+1$, where $x_{0} \in(0,1)$ is fixed. Further assume that $\left(D_{x \pm ; g}^{i \alpha} f\right)(x)=0, i=2, \ldots, n+1$. Then

$$
\begin{aligned}
\mid B_{N}^{(M)}(f)(x) & -f(x) \mid \leq \frac{(n+1) \alpha+2}{\Gamma((n+1) \alpha+2)} \\
& \times \omega_{1}\left(\left(D_{x ; g}^{(n+1) \alpha} f\right) \circ g^{-1},\left\|g^{\prime}\right\|_{\infty,[0,1]}\left(\frac{6}{\sqrt{N+1}}\right)^{\frac{1}{(n+1) \alpha+1}}\right) \\
& \times\left\|g^{\prime}\right\|_{\infty,[0,1]}^{(n+1) \alpha}\left(\frac{6}{\sqrt{N+1}}\right)^{\frac{(n+1) \alpha}{(n+1) \alpha+1}}, \quad \forall N \in \mathbb{N} .
\end{aligned}
$$

As $N \rightarrow+\infty$, we get $\left(B_{N}^{(M)}(f)\right)(x) \rightarrow f(x)$.

Proof. By Corollary 19.

REMARK 22. The truncated Favard-Szász-Mirakjan operators are given by

(60) $T_{N}^{(M)}(f)(x)=\frac{\bigvee_{k=0}^{N} s_{N, k}(x) f(k / N)}{\bigvee_{k=0}^{N} s_{N, k}(x)}, \quad x \in[0,1], N \in \mathbb{N}, f \in C_{+}([0,1])$, $s_{N, k}(x)=\frac{(N x)^{k}}{k !}($ see also [4, p. 11]). 
By [4, pp. 178-179], we get

$$
T_{N}^{(M)}(|\cdot-x|)(x) \leq \frac{3}{\sqrt{N}}, \quad \forall x \in[0,1], \forall N \in \mathbb{N}
$$

Clearly

$$
T_{N}^{(M)}\left(|\cdot-x|^{1+\beta}\right)(x) \leq \frac{3}{\sqrt{N}}, \quad \forall x \in[0,1], \forall N \in \mathbb{N}, \forall \beta>0 .
$$

The operators $T_{N}^{(M)}$ are positive sublinear operators mapping $C_{+}([0,1])$ into itself, with $T_{N}^{(M)}(1)=1$.

Furthermore

$$
\begin{aligned}
& T_{N}^{(M)}\left(|\cdot-x|^{\lambda}\right)(x)=\frac{\bigvee_{k=0}^{N} \frac{(N x)^{k}}{k !}\left|\frac{k}{N}-x\right|^{\lambda}}{\bigvee_{k=0}^{N} \frac{(N x)^{k}}{k !}}>0, \\
& \quad \forall x \in(0,1], \forall \lambda \geq 1, \forall N \in \mathbb{N} .
\end{aligned}
$$

THEOREM 23. In the setting of Theorem 21, let $x \in(0,1]$. Then

$$
\begin{aligned}
\left|T_{N}^{(M)}(f)(x)-f(x)\right| & \leq \frac{(n+1) \alpha+2}{\Gamma((n+1) \alpha+2)} \\
& \times \omega_{1}\left(\left(D_{x ; g}^{(n+1) \alpha} f\right) \circ g^{-1},\left\|g^{\prime}\right\|_{\infty,[0,1]}\left(\frac{3}{\sqrt{N}}\right)^{\frac{1}{(n+1) \alpha+1}}\right) \\
& \times\left\|g^{\prime}\right\|_{\infty,[0,1]}^{(n+1) \alpha}\left(\frac{3}{\sqrt{N}}\right)^{\frac{(n+1) \alpha}{(n+1) \alpha+1}}, \quad \forall N \in \mathbb{N} .
\end{aligned}
$$

As $N \rightarrow+\infty$, we get $\left(T_{N}^{(M)}(f)\right)(x) \rightarrow f(x)$.

Proof. By Corollary 19 .

REMARK 24. Next we study the truncated max-product Baskakov operators (see [4, p. 11])

$$
U_{N}^{(M)}(f)(x)=\frac{\bigvee_{k=0}^{N} b_{N, k}(x) f(k / N)}{\bigvee_{k=0}^{N} b_{N, k}(x)}, \quad x \in[0,1], f \in C_{+}([0,1]), N \in \mathbb{N}
$$

where

$$
b_{N, k}(x)=\left(\begin{array}{c}
N+k-1 \\
k
\end{array}\right) \frac{x^{k}}{(1+x)^{N+k}} .
$$

From [4, pp. 217-218], we get $(x \in[0,1])$

$$
\left(U_{N}^{(M)}(|\cdot-x|)\right)(x) \leq \frac{2 \sqrt{3}(\sqrt{2}+2)}{\sqrt{N+1}}, \quad \forall N \geq 2 .
$$


Let $\lambda \geq 1$. Then clearly

$$
\left(U_{N}^{(M)}\left(|\cdot-x|^{\lambda}\right)\right)(x) \leq \frac{2 \sqrt{3}(\sqrt{2}+2)}{\sqrt{N+1}}, \quad \forall N \geq 2 .
$$

Also $U_{N}^{(M)}(1)=1$, and $U_{N}^{(M)}$ are positive sublinear operators from $C_{+}([0,1])$ into itself. Furthermore

$$
U_{N}^{(M)}\left(|\cdot-x|^{\lambda}\right)(x)>0, \quad \forall x \in(0,1], \forall \lambda \geq 1, \forall N \in \mathbb{N} .
$$

Theorem 25. In the setting of Theorem 23,

$$
\begin{aligned}
\left|U_{N}^{(M)}(f)(x)-f(x)\right| \leq \frac{((n+1) \alpha+2)}{\Gamma((n+1) \alpha+2)} & \\
& \times \omega_{1}\left(\left(D_{x ; g}^{(n+1) \alpha} f\right) \circ g^{-1},\left\|g^{\prime}\right\|_{\infty,[0,1]}\left(\frac{2 \sqrt{3}(\sqrt{2}+2)}{\sqrt{N+1}}\right)^{\frac{1}{(n+1) \alpha+1}}\right) \\
& \times\left\|g^{\prime}\right\|_{\infty,[0,1]}^{(n+1) \alpha}\left(\frac{2 \sqrt{3}(\sqrt{2}+2)}{\sqrt{N+1}}\right)^{\frac{(n+1) \alpha}{(n+1) \alpha+1}}, \quad \forall N \geq 2 .
\end{aligned}
$$

As $N \rightarrow+\infty$, we get $U_{N}^{(M)}(f)(x) \rightarrow f(x)$.

Proof. By Corollary 19.

REMARK 26. Here we study the max-product Meyer-Köning and Zeller operators (see [4, p. 11]) defined by

$$
Z_{N}^{(M)}(f)(x)=\frac{\bigvee_{k=0}^{\infty} s_{N, k}(x) f\left(\frac{k}{N+k}\right)}{\bigvee_{k=0}^{\infty} s_{N, k}(x)}, \quad N \in \mathbb{N}, f \in C_{+}([0,1]),
$$

$s_{N, k}(x)=\left(\begin{array}{c}N+k \\ k\end{array}\right) x^{k}, x \in[0,1]$.

By [4, p. 253], we get

$$
Z_{N}^{(M)}(|\cdot-x|)(x) \leq \frac{8(1+\sqrt{5})}{3} \frac{\sqrt{x}(1-x)}{\sqrt{N}}, \quad \forall x \in[0,1], \forall N \geq 4, .
$$

As before we get (for $\lambda \geq 1$ )

$$
Z_{N}^{(M)}\left(|\cdot-x|^{\lambda}\right)(x) \leq \frac{8(1+\sqrt{5})}{3} \frac{\sqrt{x}(1-x)}{\sqrt{N}}:=\rho(x)
$$

for all $x \in[0,1]$ and $N \geq 4$.

Also $Z_{N}^{(M)}(1)=1$, and $Z_{N}^{(M)}$ are positive sublinear operators from $C_{+}([0,1])$ into itself. Also

$$
Z_{N}^{(M)}\left(|\cdot-x|^{\lambda}\right)(x)>0, \quad \forall x \in(0,1), \forall \lambda \geq 1, \forall N \in \mathbb{N} .
$$


TheOREM 27. In the setting of Theorem 21.

$$
\begin{aligned}
\mid Z_{N}^{(M)}(f)(x)-f & (x) \mid \leq \frac{(n+1) \alpha+2}{\Gamma((n+1) \alpha+2)} \\
& \times \omega_{1}\left(\left(D_{x ; g}^{(n+1) \alpha} f\right) \circ g^{-1},\left\|g^{\prime}\right\|_{\infty,[0,1]}(\rho(x))^{\frac{1}{(n+1) \alpha+1}}\right) \\
& \times\left\|g^{\prime}\right\|_{\infty,[0,1]}^{(n+1) \alpha}(\rho(x))^{\frac{(n+1) \alpha}{(n+1) \alpha+1}}, \quad \forall N \geq 4 .
\end{aligned}
$$

As $N \rightarrow+\infty$, we get $Z_{N}^{(M)}(f)(x) \rightarrow f(x)$.

Proof. By Corollary 19.

\subsection{Case of $(n+1) \alpha>1$}

COROLlary 28. In the setting of Theorem 16 ,

$$
\begin{aligned}
& \text { (76) }\left|L_{N}(f)\left(x_{0}\right)-f\left(x_{0}\right)\right| \leq \frac{\omega_{1}\left(\left(D_{x_{0} ; g}^{(n+1) \alpha} f\right) \circ g^{-1}, \delta\right)}{\Gamma((n+1) \alpha+1)} \\
& \times\left\|g^{\prime}\right\|_{\infty,[a, b]}^{(n+1) \alpha}\left[L_{N}\left(\left|\cdot-x_{0}\right|^{(n+1) \alpha}\right)\left(x_{0}\right)+\frac{\left\|g^{\prime}\right\|_{\infty,[a, b]} L_{N}\left(\left|\cdot-x_{0}\right|^{(n+1) \alpha+1}\right)\left(x_{0}\right)}{\delta((n+1) \alpha+1)}\right]
\end{aligned}
$$

for all $N \in \mathbb{N}$ and $\delta>0$.

Proof. Use Theorem 16 , the inequality $\left|g(x)-g\left(x_{0}\right)\right| \leq\left\|g^{\prime}\right\|_{\infty,[a, b]}\left|x-x_{0}\right|$ for all $x, x_{0} \in[a, b]$ and the properties of $L_{N}$.

Applications follow:

Theorem 29. Suppose $0<\alpha<1,(n+1) \alpha>1, n \in \mathbb{N}, f \in C_{+}([0,1])$, $f, g \in C^{1}([0,1])$, where $g$ is strictly increasing and $g^{-1} \in C^{1}([g(0), g(1)])$. Assume that $D_{x_{0}-; g}^{k \alpha} f \in C^{1}\left(\left[0, x_{0}\right]\right)$ and $D_{x_{0}+; g}^{k \alpha} f \in C^{1}\left(\left[x_{0}, 1\right]\right)$ for $k=$ $1, \ldots, n+1$, where $x_{0} \in[0,1]$ is fixed. Further assume that $\left(D_{x_{0} \pm ; g}^{i \alpha} f\right)\left(x_{0}\right)=0$, $i=2, \ldots, n+1$. Then

$$
\begin{aligned}
\mid B_{N}^{(M)}(f)\left(x_{0}\right)- & f\left(x_{0}\right) \mid \leq \frac{\omega_{1}\left(\left(D_{x_{0} ; g}^{(n+1) \alpha} f\right) \circ g^{-1},\left(\frac{6}{\sqrt{N+1}}\right)^{\frac{1}{(n+1) \alpha+1}}\right)}{\Gamma(\alpha+1)} \\
& \times\left\|g^{\prime}\right\|_{\infty,[0,1]}^{(n+1) \alpha}\left[\frac{6}{\sqrt{N+1}}+\frac{\left\|g^{\prime}\right\|_{\infty,[0,1]}\left(\frac{6}{\sqrt{N+1}}\right)^{\frac{(n+1) \alpha}{(n+1) \alpha+1}}}{(n+1) \alpha+1}\right]
\end{aligned}
$$

for all $N \in \mathbb{N}$. As $N \rightarrow+\infty$, we get $B_{N}^{(M)}(f)\left(x_{0}\right) \rightarrow f\left(x_{0}\right)$. 
Proof. By (76) we have

$$
\begin{aligned}
&\left|B_{N}^{(M)}(f)\left(x_{0}\right)-f\left(x_{0}\right)\right| \leq \frac{\omega_{1}\left(\left(D_{x_{0} ; g}^{(n+1) \alpha} f\right) \circ g^{-1}, \delta\right)}{\Gamma((n+1) \alpha+1)}\left\|g^{\prime}\right\|_{\infty,[0,1]}^{(n+1) \alpha} \\
& \times\left[B_{N}^{(M)}\left(|\cdot-x|^{(n+1) \alpha}\right)\left(x_{0}\right)+\frac{\left\|g^{\prime}\right\|_{\infty,[0,1]} B_{N}^{(M)}\left(\left|\cdot-x_{0}\right|^{(n+1) \alpha+1}\right)\left(x_{0}\right)}{\delta((n+1) \alpha+1)}\right] \\
& \leq \frac{\omega_{1}\left(\left(D_{x_{0} ; g}^{(n+1) \alpha} f\right) \circ g^{-1}, \delta\right)}{\Gamma((n+1) \alpha+1)}\left\|g^{\prime}\right\|_{\infty,[0,1]}^{(n+1) \alpha} \\
& \times\left[\frac{6}{\sqrt{N+1}}+\frac{\left\|g^{\prime}\right\|_{\infty,[0,1]} \frac{6}{\sqrt{N+1}}}{\delta((n+1) \alpha+1)}\right]=:(*) .
\end{aligned}
$$

Choose $\delta=\left(\frac{6}{\sqrt{N+1}}\right)^{\frac{1}{(n+1) \alpha+1}}$, then $\delta^{(n+1) \alpha+1}=\frac{6}{\sqrt{N+1}}$, and apply it to 78 . We get

$$
\begin{aligned}
(*)= & \frac{\omega_{1}\left(\left(D_{x_{0} ; g}^{(n+1) \alpha} f\right) \circ g^{-1},\left(\frac{6}{\sqrt{N+1}}\right)^{\frac{1}{(n+1) \alpha+1}}\right)}{\Gamma((n+1) \alpha+1)}\left\|g^{\prime}\right\|_{\infty,[0,1]}^{(n+1) \alpha} \\
& \times\left[\frac{6}{\sqrt{N+1}}+\frac{\left\|g^{\prime}\right\|_{\infty,[0,1]} \delta^{(n+1) \alpha+1}}{\delta((n+1) \alpha+1)}\right] \\
= & \frac{\omega_{1}\left(\left(D_{x_{0} ; g}^{(n+1) \alpha} f\right) \circ g^{-1},\left(\frac{6}{\sqrt{N+1}}\right)^{\frac{1}{(n+1) \alpha+1}}\right)}{\Gamma((n+1) \alpha+1)}\left\|g^{\prime}\right\|_{\infty,[0,1]}^{(n+1) \alpha} \\
& \times\left[\frac{6}{\sqrt{N+1}}+\frac{\left\|g^{\prime}\right\|_{\infty,[0,1]}\left(\frac{6}{\sqrt{N+1}}\right)^{\frac{(n+1) \alpha}{(n+1) \alpha+1}}}{(n+1) \alpha+1}\right]
\end{aligned}
$$

for all $N \in \mathbb{N}$, proving the claim.

TheOrem 30. In the setting of Theorem 29,

$$
\begin{aligned}
\mid T_{N}^{(M)}(f)\left(x_{0}\right) & -f\left(x_{0}\right) \mid \leq \frac{\omega_{1}\left(\left(D_{x_{0} ; g}^{(n+1) \alpha} f\right) \circ g^{-1},\left(\frac{3}{\sqrt{N}}\right)^{\frac{1}{(n+1) \alpha+1}}\right)}{\Gamma((n+1) \alpha+1)} \\
& \times\left\|g^{\prime}\right\|_{\infty,[0,1]}^{(n+1) \alpha}\left[\frac{3}{\sqrt{N}}+\frac{\left\|g^{\prime}\right\|_{\infty,[0,1]}}{(n+1) \alpha+1}\left(\frac{3}{\sqrt{N}}\right)^{\frac{(n+1) \alpha}{(n+1) \alpha+1}}\right]
\end{aligned}
$$

for all $N \in \mathbb{N}$. We get $\lim _{N \rightarrow+\infty} T_{N}^{(M)}(f)\left(x_{0}\right)=f\left(x_{0}\right)$. 
TheOREM 31. In the setting of Theorem 29,

$$
\begin{aligned}
\left|U_{N}^{(M)}(f)\left(x_{0}\right)-f\left(x_{0}\right)\right| & \\
\leq & \frac{\omega_{1}\left(\left(D_{x_{0} ; g}^{(n+1) \alpha} f\right) \circ g^{-1},\left(\frac{2 \sqrt{3}(\sqrt{2}+2)}{\sqrt{N+1}}\right)^{\frac{1}{(n+1) \alpha+1}}\right)\left\|g^{\prime}\right\|_{\infty,[0,1]}^{(n+1) \alpha}}{\Gamma((n+1) \alpha+1)} \\
& \times\left[\frac{2 \sqrt{3}(\sqrt{2}+2)}{\sqrt{N+1}}+\frac{\left\|g^{\prime}\right\|_{\infty,[0,1]}}{(n+1) \alpha+1}\left(\frac{2 \sqrt{3}(\sqrt{2}+2)}{\sqrt{N+1}}\right)^{\frac{(n+1) \alpha}{(n+1) \alpha+1}}\right]
\end{aligned}
$$

for all $N \geq 2$. We get $\lim _{N \rightarrow+\infty} U_{N}^{(M)}(f)\left(x_{0}\right)=f\left(x_{0}\right)$.

Proof. Similar to Theorem 29 .

THEOREM 32. In the setting of Theorem 29.

$$
\begin{aligned}
\mid Z_{N}^{(M)}(f)\left(x_{0}\right)- & f\left(x_{0}\right) \mid \\
\leq & \frac{\omega_{1}\left(\left(D_{x_{0} ; g}^{(n+1) \alpha} f\right) \circ g^{-1},(\rho(x))^{\frac{1}{(n+1) \alpha+1}}\right)\left\|g^{\prime}\right\|_{\infty,[0,1]}^{(n+1) \alpha}}{\Gamma((n+1) \alpha+1)} \\
& \times\left[\rho(x)+\frac{\left\|g^{\prime}\right\|_{\infty,[0,1]}}{(n+1) \alpha+1}(\rho(x))^{\frac{(n+1) \alpha}{(n+1) \alpha+1}}\right],
\end{aligned}
$$

for all $N \geq 4$, and $\lim _{N \rightarrow+\infty} Z_{N}^{(M)}(f)\left(x_{0}\right)=f\left(x_{0}\right)$, where $\rho(x)$ is as in 73 .

Proof. Similar to Theorem 29 .

Corollary 33 (to Theorem 29p. Let $0<\alpha<1$, and $(n+1) \alpha>1$, $n \in \mathbb{N}, f \in C_{+}([0,1])$ and $f \in C^{1}([0,1])$. Assume that $D_{x_{0}-; e^{x}}^{k \alpha} f \in C^{1}\left(\left[0, x_{0}\right]\right)$ and $D_{x_{0}+e^{x}}^{k \alpha} f \in C^{1}\left(\left[x_{0}, 1\right]\right)$ for $k=1, \ldots, n+1$, where $x_{0} \in[0,1]$ is fixed. Further assume that $\left(D_{x_{0} \pm ; e^{x}}^{i \alpha} f\right)\left(x_{0}\right)=0, i=2, \ldots, n+1$. Then

$$
\begin{aligned}
\mid B_{N}^{(M)}(f)\left(x_{0}\right)- & f\left(x_{0}\right) \mid \\
\leq & \frac{\omega_{1}\left(\left(D_{x_{0} ; e^{x}}^{(n+1) \alpha} f\right) \circ \ln t,\left(\frac{6}{\sqrt{N+1}}\right)^{\frac{1}{(n+1) \alpha+1}}\right) e^{(n+1) \alpha}}{\Gamma((n+1) \alpha+1)} \\
& \times\left[\frac{6}{\sqrt{N+1}}+\frac{e\left(\frac{6}{\sqrt{N+1}}\right)^{\frac{(n+1) \alpha}{(n+1) \alpha+1}}}{(n+1) \alpha+1}\right], \quad \forall N \in \mathbb{N} .
\end{aligned}
$$

We have $\lim _{N \rightarrow+\infty} B_{N}^{(M)}(f)\left(x_{0}\right)=f\left(x_{0}\right)$.

Proof. Use Theorem 29 with $g(x)=e^{x}$. 


\section{References}

[1] G. Anastassiou, Advanced fractional Taylor's formulae, J. Comput. Anal. Appl. 21 (2016), 1185-1204.

[2] G. Anastassiou, Nonlinearity: Ordinary and Fractional Approximations by Sublinear and Max-Product Operators, Springer, Heidelberg, 2018.

[3] G. Anastassiou and I. Argyros, Intelligent Numerical Methods: Applications to Fractional Calculus, Springer, Heidelberg, 2016.

[4] B. Bede, L. Coroianu and S. Gal, Approximation by Max-Product Type Operators, Springer, Heidelberg, 2016.

[5] H. L. Royden, Real Analysis, 2nd ed., Macmillan, New York, 1968.

George A. Anastassiou

Department of Mathematical Sciences

University of Memphis

Memphis, TN 38152, U.S.A.

E-mail: ganastss@memphis.edu 
